\title{
Effective Parameters for the Precise Control of Thin Film Buckling on Elastomeric Substrates
}

\author{
Seong-Min Ahn and Chang-Hyun Jang* \\ College of BioNano Technology, Kyungwon University, Seongnam, Gyeonggi-Do 461-701, Korea \\ ${ }^{*}$ E-mail: chjang4u@kyungwon.ac.kr \\ Received October 6, 2009, Accepted January 6, 2010
}

\begin{abstract}
This paper reports a simple and versatile technique for generating highly controllable sinusoidal nanostructures on the surface of poly-(dimethylsiloxane) (PDMS). The sinusoidal features were generated by oxidizing PDMS slabs with oxygen plasma, then stretching them by wrapping around a cylinderical surface, and finally allowing them to relax. The wavelength and amplitude could be finely controlled by varying the fabrication conditions such as duration of oxidation, diameter of the glass cylinder, duration of stretching, thickness of the PDMS slabs, and temperature during the second hardening process. The varied trends of the buckling patterns were characterized by using an atomic force microscope.
\end{abstract}

Key Words: Buckling pattern, Poly-(dimethylsiloxane), Oxygen plasma, Wavelength, Amplitude

\section{Introduction}

Polymeric soft substrates with periodic nanostructures have the potential to be important components of many emerging technologies, including paper-like displays, ${ }^{1}$ electronic eyes, ${ }^{2}$ conformable skin sensors, ${ }^{3}$ smart surgical gloves, ${ }^{4}$ and health monitoring devices, ${ }^{5}$ due to their ease of processing, mechanical flexibility, and highly controllable physicochemical properties. It has been reported that thin film contraction under external mechanical stress can be used to create periodic patterned features on the surface of thin silica or metal films on elastic substrates. ${ }^{6-9}$ Buckling of stiff thin films on a compliant substrate such as poly-(dimethylsiloxane) (PDMS) was first observed in the late $1990 \mathrm{~s}$, where the stiff thin films were attached to the unembellished surface of a compliant substrate that was subject to prestain. ${ }^{10}$ Relaxation of the pre-strain in the compliant substrate led to the buckling of the stiff thin films, which produced highly periodic, sinusoidal wave patterns. Although buckling is considered a mechanical failure on the surface, the pioneering work by Bowden et al. ${ }^{10}$ in 1998 demonstrated that this behavior can be controlled on the sub-micrometer scale to generate interesting structures with well defined geometries and dimensions. These observations have attracted huge interest in this area and many scientists are currently exploring the basic scientific aspects ${ }^{11-14}$ of this process as well as practical applications. ${ }^{15-18}$ However, mechanical instability of soft nanostructures can be an issue when the dimensions of the periodic structures are reduced to the nanometer scale. ${ }^{19-21}$ Thus, the development of methods to selectively produce an intended mode of the nanostructure is desired, where both structural integrity and material flexibility are ensured.

The main purpose of this work is to elucidate that there are direct connections between the dimensions of the buckling waves and the fabrication conditions. We investigate the effects of different fabrication parameters such as oxidation time, diameter of cylinder, stretching time, PDMS slab thickness, and stretching temperature on the dimensions of the thin film buckling.

\section{Experimental Details}

Procedure for the buckling of polymer thin films. Flat PDMS (Sylgard 184; Dow Corning) substrates were prepared by mixing siloxane base resin and crosslinking agent (10:1 weight ratio), and casted against a polystyrene Petri dish (10 cm in diameter). They were left at low pressure to allow trapped air bubbles to escape from the mixture and then cured overnight at 80 ${ }^{\circ} \mathrm{C}$. The PDMS was cut into $4 \mathrm{~cm} \times 1 \mathrm{~cm}$ slabs and exposed to oxygen plasma (Covance, Femto Science) at $150 \mathrm{~W}$ with a flow rate of $20 \mathrm{sccm}$ to generate a thin, silica-like film on the surface of the substrate. The plasma-treated PDMS was then wrapped around a cylindrical surface. The sinusoidal wave patterns were generated within the brittle layer of the PDMS upon relaxation of the strain.

Characterization of buckling patterns. The surface topology of the PDMS substrates after treatment was characterized by using a tapping-mode atomic force microscope (NanoScope IIIa, Veeco Metrology, Santa Barbara, CA). The wavelength and the amplitude of the buckling patterns were determined by taking an average of the peak-to-peak and peak-to-trough distance, respectively, of 10 parallel waves.

\section{Results and Discussion}

In this study, an experimentally straightforward technique was developed to generate a highly controlled parallel array of waves on an elastic substrate. A PDMS slab was first oxidized by oxygen plasma, wrapped around a glass cylinder, and then unwrapped. Due to the difference in the expansion and restoring force (elastic coefficient) between the oxidized film and the bulk substrate, continuous wave patterns were generated by relieving the stress generated during expansion. The dimensions of the waves were reproducibly and uniformly generated when the experimental conditions affecting the wave characteristics were quantitatively controlled. The experimental conditions that could be used to reliably control the dimensions of the sinusoidal 


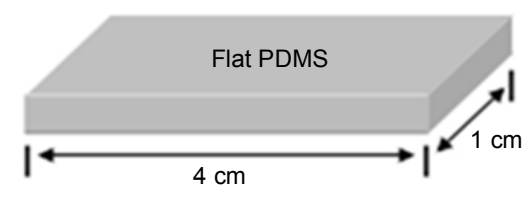

$\downarrow$ 1. Oxygen plasma
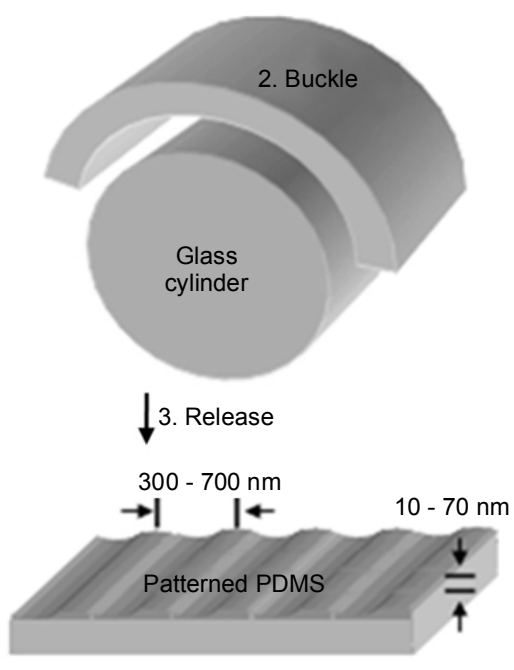

Figure 1. Schematic illustration of the steps used to precisely control thin film buckling of the PDMS substrate.

waves were duration of oxidation of the PDMS substrate, diameter of the glass cylinder, duration of stretching, thickness of the PDMS slab, and temperature during the second hardening process. Sinusoidal waves with an average wavelength between $300 \mathrm{~nm}$ and $800 \mathrm{~nm}$ and an amplitude between $10 \mathrm{~nm}$ and $70 \mathrm{~nm}$ were reproducibly generated by controlling the fabrication conditions. We then demonstrated that there were direct connections between the dimension of waves and the fabrication parameters described above.

Figure 1 schematically illustrates the steps used to form parallel array of waves on the surface of oxidized PDMS. The procedure begins with curing the flat PDMS (Sylgard 184), which was prepared by mixing a 10:1 ratio of pre-polymer and curing agent, then allowing the PDMS mixture to cure overnight at $80^{\circ} \mathrm{C}$. The hardened PDMS slab, which had a typical dimension of $4 \mathrm{~cm} \times 1 \mathrm{~cm} \times 0.35 \mathrm{~cm}$, was exposed to oxygen plasma at 150 $\mathrm{W}$ to generate a thin, silica-like film on the surface of the substrate. The plasma-treated PDMS was then wrapped around a cylindrical surface. The sinusoidal wave patterns were generated within the brittle layer of the PDMS in a direction perpendicular to that of the applied strain upon relaxation of the strain. We then evaluated the wavelength and amplitude of the surface topography under a wide range of different fabrication conditions, which were shown to affect the resulting dimensions of the sinusoidal wave pattern.

The topography of the surface was examined using a tapping-mode atomic force microscope (AFM). Representative images of the wave features are shown in Figure 2. Cross-sectional images of the wave features confirmed that they had a regular periodicity and amplitude. The wave features for all pat-

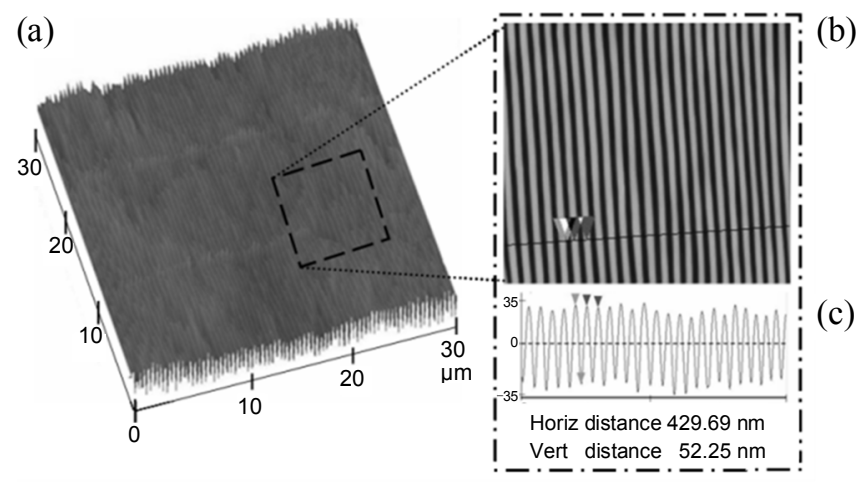

Figure 2. Tapping-mode AFM image of a 2D wavy nanostructure on PDMS. (a) Three-dimensional image within a relatively large area, $30 \mu \mathrm{m} \times 30 \mu \mathrm{m}$, (b) Magnified image of the area indicated by a small square $(10 \mu \mathrm{m} \times 10 \mu \mathrm{m})$ in Fig. (a) with a cross-sectional line profile showing the 1D wavy characteristics of the surface.

terns were nearly sinusoidal. The wavelength ranged from $300 \mathrm{~nm}$ to $800 \mathrm{~nm}$ and the amplitude ranged from $10 \mathrm{~nm}$ to $70 \mathrm{~nm}$ depending on the experimental conditions. The patterns were well ordered over distances of $\sim 1 \mathrm{~cm}$.

Figure 3 shows the wave characteristics relative to the fabrication conditions. Figure $3 \mathrm{~A}$ demonstrates that the wavelength and the amplitude of the waves can be controlled by varying the duration of oxidation. The hardened PDMS slab, which had a dimension of $4 \mathrm{~cm} \times 1 \mathrm{~cm} \times 0.35 \mathrm{~cm}$, was exposed to an oxygen plasma (COVANCE, Femto Science) at $150 \mathrm{~W}$ with a flow rate of $20 \mathrm{sccm}$ and and a base pressure of 0.9 Torr, for different exposure times ( $2 \mathrm{~min}, 4 \mathrm{~min}, 6 \mathrm{~min}$, and $8 \mathrm{~min}$ ). The plasmatreated PDMS was then wrapped around a cylindrical surface that had a diameter of $5 \mathrm{~cm}$ for $2 \mathrm{~h}$. The sinusoidal wave patterns were evaluated by AFM after relaxation of the strain. As shown in Figure 3A, the wavelength and amplitude increased in proportion to the exposure time to oxygen plasma. It has been known that the thickness of the silica-like thin film increases with the duration of plasma treatment. ${ }^{6}$ An increase in the film thickness reduces the flexibility of the film, and, therefore, increases the periodicity of waves. On the other hand, the built-up compressive stress at an increased wavelength is relieved in a direction perpendicular to the plane of surface, which results in an increase in the amplitude of the waves. However, there is a practical limit as to how large the amplitude and wavelength can be increased through increased oxidation. Since oxidized PDMS is brittle, when the surface is oxidized excessively $(>10$ min), crack defects become more common.

Figure $3 \mathrm{~B}$ shows that the diameter of the glass cylinder can be used as an effective parameter to control the buckling patterns. When the PDMS was wrapped around a cylinder with a shorter diameter, the difference in the amount of stretching between the outer and inner layer becomes greater. This results in a higher misfit of restoring force between the oxidized film and the bulk substrate upon release from the cylinder. Therefore, the periodicity of the wave decreases and the amplitude increases to relieve the built-up compressive stress in the oxidized film.

We also evaluated the dependence of the wave characteristics on stretching time (Figure 3C). As the time over which the 
(a)

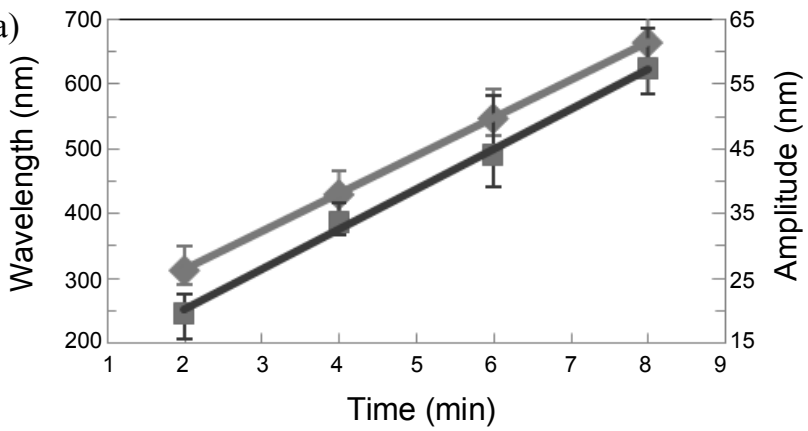

(c)
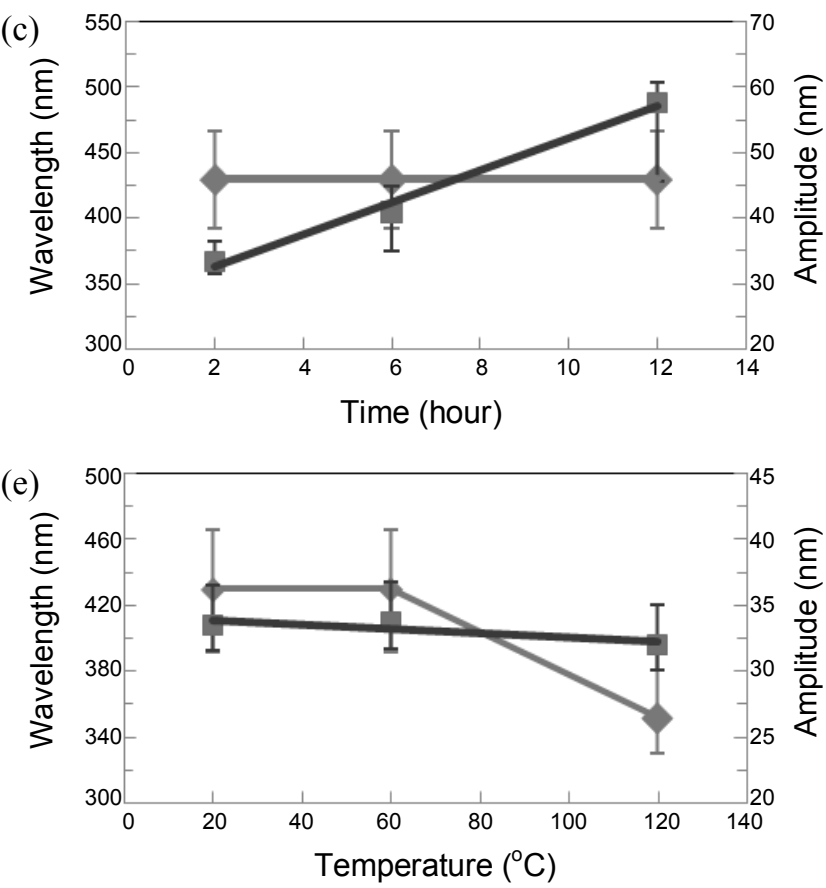
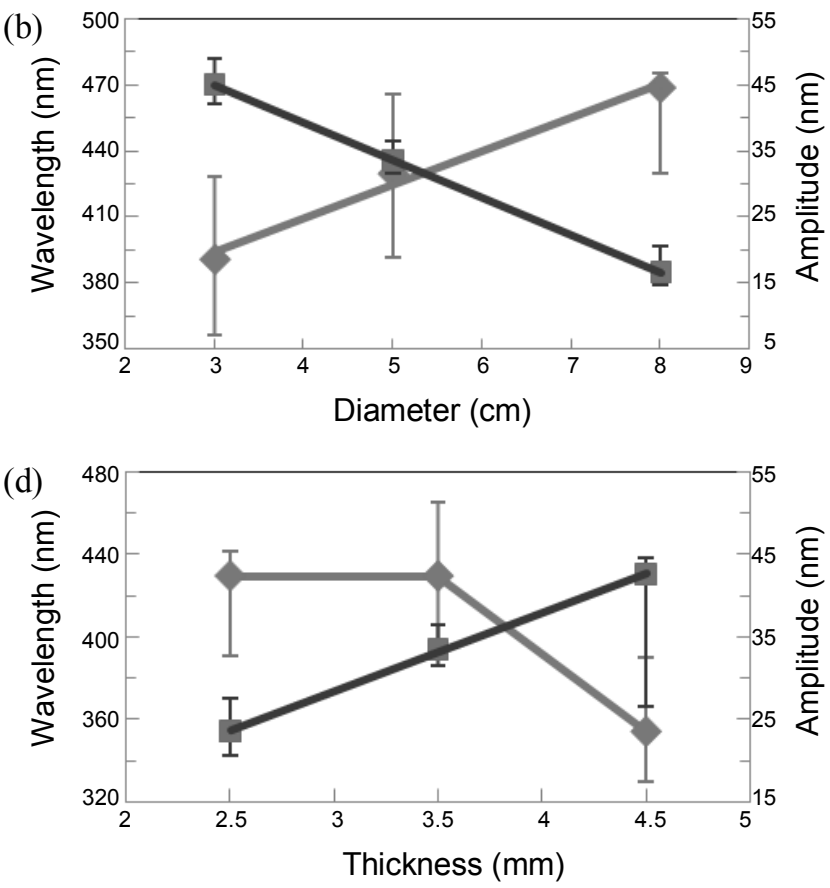

Wavelength Amplitude

Figure 3. Dependence of wavelength and amplitude of the wave features on fabrication conditions: (a) duration of oxidation, (b) diameter of the glass cylinder, (c) duration of stretching, (d) thickness of the PDMS substrate, (e) second hardening temperature.

PDMS slab was subjected to mechanical stress increased, the restoring force of the oxidized film gradually decreased. When released from the cylinder, the bulk PDMS returned approximately to its original shape; thus, the restoration of the oxidized film was inversely proportional to the duration of stretching. As a result, longer stretching time resulted in a greater amplitude of the waves. In the fabrication conditions tested in this study, only a small decrease in wavelength was observed.

The dependence of the wave characteristics on the thickness of the PDMS substrates was displayed in Figure 3D. As the thickness of the PDMS substrate increased, the difference in the amount of stretching between the outer and inner layer became greater while wrapped around the cylindrical surface. Therefore, the use of thicker substrates induced a higher misfit strain at the interface between the oxidized film and the bulk PDMS upon release from the cylinder, which resulted in a decrease in the buckling wavelength and an increase in amplitude.

Finally, we evaluated the dependence of the wave characteristics on the stretching temperature (Figure 3E). The plasma-treated PDMS was wrapped around a cylindrical surface with a diameter of $5 \mathrm{~cm}$ and then heated at three different temperatures $\left(20{ }^{\circ} \mathrm{C}, 60{ }^{\circ} \mathrm{C}\right.$, and $\left.120{ }^{\circ} \mathrm{C}\right)$ in a convection oven. After $2 \mathrm{~h}$ of heating at each designated temperature, the PDMS slabs were released from the cylinder and allowed to cool down to room temperature. There was no change in wavelength observed between $20^{\circ} \mathrm{C}$ and $60^{\circ} \mathrm{C}$. However, we observed a significant decrease in the buckling wavelength when the PDMS was stretched at $120^{\circ} \mathrm{C}$. We attributed the reduced wavelength to a further decrease in the restoring force of the oxidized film while heated at high temperature. No change in amplitude was observed under these experimental conditions.

\section{Conclusion}

We have described a simple and convenient method to fabricate surfaces patterned with a continuous array of sinusoidal waves. We also demonstrated that there were direct connections between the dimensions of the buckling waves and the fabrication conditions. The effects of different parameters such as oxidation time, diameter of cylinder, stretching time, PDMS slab thickness, and stretching temperature were investigated. The results presented here might provide useful tools for the inte- 
gration of stretchable electronics and health monitoring devices. We also believe that the versatility of this technology will be useful in fundamental studies such as examining the interaction between cells and artificial materials.

Acknowledgments. This work was supported by the Kyungwon University Research Fund in 2009 and the GRRC program of Gyeonggi province (Grant No. GRRC Kyungwon 2009A02).

\section{References}

1. Rogers, J. A.; Bao, Z.; Baldwin, K.; Dodabalapur, A.; Crone, B.; Raju, V. R.; Kuck, V.; Katz, H.; Amundson, K.; Ewing, J.; Drzaic, P. Proc. Natl. Acad. Sci. USA 2001, 98, 4835.

2. Jin, H. C.; Abelson, J. R.; Erhardt, M. K.; Nuzzo, R. G. J. Vac. Sci. Technol. 2004, B22, 2548.

3. Lumelsky, V.; Shur, M. S.; Wagner, S. IEEE Sens. J. 2001, 1, 41.

4. Someya, T.; Sekitani, T.; Iba, S.; Kato, Y.; Kawaguchi, H.; Sakurai, T. Proc. Natl. Acad. Sci. USA 2004, 101, 9966.

5. Nathan, A.; Park, B.; Sazonov, A.; Tao, S.; Chan, I.; Servati, P.; Karim, K.; Charania, T.; Striakhilev, D.; Ma, Q.; Murthy, R. V. R. Microelectron J. 2000, 31, 883

6. Chua, D. B. H.; Ng, H. T. Appl. Phys. Lett. 2000, 76, 721.

7. Sun, Y.; Choi, W. M.; Jiang, H.; Huang, Y.; Rogers, J. A. Nat. Nanotechnol. 2006, 1, 201.
8. Jiang, X.; Takayama, S.; Qian, X.; Ostuni, E.; Wu, H.; Bowden, N.; LeDuc, P.; Ingber, D. E.; Whitesides, G. M. Langmuir 2002, 18, 3273.

9. Lam, M. T.; Sim, S.; Zhu, X.; Takayama, S. Biomaterials 2006, 27, 4340.

10. Bowden, N.; Brittain, S.; Evans, A. G.; Hutchinson, J. W.; Whitesides, G. M. Nature 1998, 393, 146.

11. Harris, A. K.; Wild, P.; Stopak, D. Science 1980, 208, 177.

12. Moon, M. W.; Lee, S. H.; Sun, J. Y.; Oh, K. H.; Vaziri, A.; Hutchinson, J. W. Proc. Natl. Acad. Sci.USA 2007, 104, 1130.

13. Sharp, J. S.; Jones, R. A. L. Adv. Mater. 2002, 14, 799.

14. Schmid, H.; Wolf, H.; Allenspach, R.; Riel, H.; Karg, S.; Michel, B.; Delamarche, E. Adv. Funct Mater. 2003, 13, 145.

15. Khang, D. Y.; Jiang, H. Q.; Huang, Y.; Rogers, J. A. Science 2006, $311,208$.

16. Stafford, C. M.; Harrison, C.; Beers, K. L.; Karim, A.; Amis, E. J.; Vanlandingham, M. R.; Kim, H. C.; Volksen, W.; Miller, R. D.; Simonyi, E. E. Nat. Mater. 2004, 3, 545.

17. Efimenko, K.; Rackaitis, M.; Manias, E.; Vaziri, A.; Mahadevan, L.; Genzer, J. Nat. Mater. 2005, 4, 293.

18. Wilder, E. A.; Guo, S.; Lin-Gibson, S.; Fasolka, M. J.; Stafford, C. M. Macromolecules 2006, 39, 4138.

19. Bakajin, G.; Fountain, E.; Morton, K.; Chou, S. Y.; Sturm, J. C.; Austin, R. H. MRS Bull. 2006, 31, 108.

20. Hsueh, C. H.; Lee, S.; Lin, H. Y.; Chen, L. S.; Wang, W. H. Mater. Sci. Eng. A 2006, 433, 316.

21. Chuang, W. C.; Ho, C. T.; Wang, W. C. Opt. Express 2005, 13, 6685 . 